\title{
LIMITS ON THE VARIATION OF PHYSICAL CONSTANTS DERIVED FROM MOLECULAR ABSORPTION LINES
}

\author{
T. WIKLIND
}

Onsala Space Observatory, S-43992 Onsala, Sweden

\author{
AND \\ F. COMBES \\ Observatoire de Paris \\ 61 Av. de l'Observatoire, F-75014 Paris, France
}

A potential diagnostic application of molecular rotational absorption lines at high redshift is to test the invariance of physical constants. This can be done by comparing the observed redshifted frequency of a molecular absorption line with redshifted lines from other types of transitions such as the $21 \mathrm{~cm}$ hyperfine transition or electronic resonance transitions. In order to set stringent limits, it is necessary to achieve the greatest possible frequency resolution. This makes radio lines well suited for this purpose.

The frequency of a rotational transition of a linear molecule is, to first order $\nu_{\text {rot }} \propto m_{\mathrm{e}}^{2} \alpha^{2} / \mu$, where $\mu$ is the reduced mass of the molecule. For the $21 \mathrm{~cm}$ hyperfine transition of atomic hydrogen the frequency is $\nu_{h f} \propto$ $m_{\mathrm{e}}^{2} \alpha^{4} g_{p} / m_{\mathrm{p}}$. Hence the ratio of the transition frequencies is $\propto \alpha^{2} g_{p}\left(\mu / m_{\mathrm{p}}\right)$. $m_{\mathrm{e}}$ and $m_{\mathrm{p}}$ are the electron and proton masses, respectively. A comparison of molecular rotational transitions with the $21 \mathrm{~cm}$ hyperfine transition therefore tests the invariance of the proton gyromagnetic ratio $g_{p}$ and the fine structure constant $\alpha$. The frequency of a molecular rotational transition is affected by centrifugal stretching, allowing a comparison of different transitions from the same molecule, or rotational lines from different molecules. Here it is the invariance of the nucleon masses that is tested. At $\mathrm{z}=0.25 \&$ $0.68, \Delta z /(1+z)<10^{-5}$, at $3 \sigma$, or $<2 \times 10^{-15}$ year $^{-1}$, when comparing molecular and $21 \mathrm{~cm}$ transitions. The main limitation to the use of radio lines is that different transitions may probe different gas volumes along the line of sight, with a possible velocity difference. This is true also when comparing only rotational transitions, as the opacity depends on the excitation conditions, which can vary along the line of sight. Hence, any value set by comparisons of these types must be regarded with caution. 